\title{
Studies of Preservative and Coloring Agent in Jam and Jelly
}

\author{
Salma Akter Mou, Tanzina Akther, Mohammad Shoeb*, Abida Sultana and Nilufar Nahar \\ Department of Chemistry, Dhaka University, Dhaka-1000, Bangladesh
}

(Received: 26 October 2016; Accepted: 12 December 2016)

\section{Introduction}

Jam and Jelly are popular processed foods in which food additives are added ${ }^{1}$. Preservatives are added to jam and jelly for preserving, increasing shelf-life and inhibiting the growth of pathogens ${ }^{2}$. Colorings agents are added to enhance original fruit color and to make lucrative.

Sodium benzoate is an antimicrobial agent and used as preservatives in jam, jelly, soft drinks, beverages, cosmetics even in medicines. The US food and Drug Administration (FDA) and the Canadian Health Protection Branch have told sodium benzoate as preservative to be acceptable when its limit in food is $0.1 \%$ by weight. Daily intake of sodium benzoate higher than acceptable limit can bring adverse effects on skin such as rash, non-immunological contact urticarial, metabolic acidosis, hyperpnoea and asthma ${ }^{3,4}$. Sodium benzoate may decarboxylate after consumption and produces benzene in human body which is carcinogenic.

Yellow 6 is used as a colorant food additive in many food products such as apricot jam, custard powders, citrus marmalade, orange soft drinks, sweets, squashes, margarine, marzipan, packet soups, ice-creams etc ${ }^{5}$. According to European Food Safety Authority (EFSA) acceptable daily intake (ADI) for sunset yellow is $1.0 \mathrm{mg} / \mathrm{kg}$ bodyweight per day. Above ADI it can induces allergic reaction, diarrhea, migraines, gastric upset and swelling of the skin, nettle rash vomiting etc ${ }^{5}$.

\section{Experimental}

\section{Preparation of standard solutions}

The primary standard solution of sodium benzoate and yellow $6(100 \mathrm{mg} / \mathrm{L})$ was prepared in Milli-Q water which was free from ions. The working standard solutions were prepared in concentration 75.0, 50.0, 20.0, 10.0, 5.0, 4.0, and $2.0 \mathrm{mg} / \mathrm{L}$ from primary standard solution by dilution with water. Absorbances of these solutions were measured by a double beam UV-visible spectrophotometer at 225 and $484 \mathrm{~nm}$ for sodium benzoate and yellow 6 respectively and calibration curves were made.

\section{Preparation of samples}

Jam or jelly samples $(5.0 \mathrm{~g})$ were taken to obtain $250 \mathrm{mg} / \mathrm{L}$ solution which was vortexed for $30 \mathrm{sec}$ and centrifuged. Then the solution was filtered and diluted to obtain $25 \mathrm{mg} / \mathrm{L}$ solution. The absorbances of the prepared solutions were measured at 225 and $484 \mathrm{~nm}$ from which sodium benzoate and yellow 6 content were determined respectively.

\section{Determination of total carbohydrate}

Total carbohydrate in juice solutions were determined performing modified Molisch's method and using the standard calibration curve made for glucose ${ }^{6}$.

\section{Determination of $\mathrm{pH}$ value}

$\mathrm{pH}$ of each sample was determined using Microprocessor pH meter (Model: HANNA pH 211).

\section{Results and Discussion}

Analysis of samples

Analyses of sodium benzoate and yellow 6 were carried out in 27 samples by UV-visible spectrophotometric method. Among jam samples five samples contained sodium benzoate in the range of $254.5-400.4 \mathrm{mg} / \mathrm{kg}$ (i.e. $0.25 \%$ $0.40 \%$ ). All jelly samples were found to contain sodium benzoate in a range of $160-289 \mathrm{mg} / \mathrm{kg}$, (approximately $0.16 \%$ to $0.28 \%$ ). The allowable limit of sodium benzoate is $0.1 \%$ in jelly $^{7}, 8$. Out of 15 jelly samples 10 samples contained yellow 6 in the range of $0.011 \%$ to $0.014 \%$. However, yellow 6 was not found in any jam samples. According to Health Canada, 2015, the allowable limit of yellow 6 in food is $0.3 \%$ by weight 9 .

\section{Total carbohydrate}

Carbohydrate content of commercial brand jelly and jam samples is in the range of 50.72-84 g/kg and $9.0-17.0 \mathrm{~g} / \mathrm{kg}$ respectively. High carbohydrates contained jelly causes obesity to the consumers ${ }^{10}$.

$p H$

Due to the presence of naturally occurring organic acid, orange juices usually are acidic and have $\mathrm{pH}$ around $3.5^{6}$. $\mathrm{pH}$ of commercial brand orange jelly and mixed fruit jam samples were found to be in the range of 2.65-2.87 and 2.65-2.87 respectively. A lower $\mathrm{pH}$ might be due to the presence of acidic additives which can cause acidity in the stomach and erosion of the tooth ename ${ }^{11}$. The recovery of the used procedure, which reflects the accuracy of the analytical method, was evaluated by analyzing preservative free sample spiked with $10 \mathrm{mg} / \mathrm{L}$ sodium benzoate. The mean recovery of ten measurements obtained by standard addition approach was found to be 110.04 with relative standard deviation $2.40 \%$.

\section{Conclusions}

Although the number of samples analyzed is still small, the data presented in this study gave a preliminarily outline about the content of food additives in jam and jelly frequently consumed in Bangladesh. Based on these analytical data, it seems that the content levels of yellow 6

\footnotetext{
*Author for correspondence. e-mail: shoeb71@yahoo.com
} 
are within the maximum authorized levels. However, sodium benzoate content exceeded the limit.

\section{Acknowledgement}

The authors are grateful to International Science Programme (ISP), Uppsala, Sweden and Higher Education Quality Enhancement Project (HEQEP), UGC, Dhaka for financial supports.

\section{References}

1. Nazim, S., M. Farooqui, S. S. Kasim, 2016. Analysis of physicochemical properties and preservatives in jams. $J$. Med Chem and Drug Disc, 2 (1), 517-522.

2. International Food Information Council (IFIC) and U.S. Food and Drug Administration, May 23, 2011. Food Ingredients and Colors.

3. Bronaugh, R. L., R. F. Stewart and E. R. Congdon, 1982. Methods for in vitro percutaneous absorption studies II animal methods for human skin. J. Tox and App Pharm, 62, 481-488.

4. Coverly, J., L. Peters, E. Whittle, and D. A. Basketter, 1998. Susceptibility to skin stinging, non-immunologic contact urticarial and acute skin irritation. Contact Dermatitis 38, 90-95.

5. Kus, E. and H. E. Eroglu, 2015. Genotoxic and cytotoxic effects of sunset yellow and brilliant blue, colorant food additives, on human blood lymphocytes. Pak J. pharm sci. 28 (1), 227.
6. Sultana, A., M. S. Haque, M. Shoeb, M. S. Islam, M. I. R. Mamun and N. Nahar, 2012. Presence of yellow 6, an artificial colour additive in orange juice, JBCS, 25 (1), 80-86.

7. Sultana, A., R. Islam, Md. M. Islam, M. Shoeb and N. Nahar, 2016. Study of preservatives and stimulants in commercial soft drinks, Bangladesh Pharm J. 19 (1), 68-74.

8. Vivekreddy, M., G. Aruna, S. Angalaparameswari, B. Haseenabanu, P. Jayachandrareddy, 2015. Estimated daily intake and exposure of sodium benzoate and potassium sorbate through food products in school children of tirupati, india, Int J. Pharm and Pharm Sci. 7, Issue 7.

9. Health Canada, 2015, List of Permitted Food Additives.

10. Malik, V. S., M. B. Schulze; Hu, F B 2006. Intake of sugarsweetened beverages and weight gain, Amer J. Cli Nut 84 (2), 274-28.

11. Wongkhantee, S., V. Patanapiradej, C. Maneenut, D. Tantbirojn, 2005. Dental erosion and severe tooth decay related to soft drinks, J. Dentistry 34 (3), 1-7. 